\title{
Off the Beaten Path: Almost Clean Formation of Indene from the ortho-Benzyne + Allyl Reaction
}

\author{
Morgan N. McCabe, Patrick Hemberger, Engelbert Reusch, Andras Bodi, and Jordy Bouwman*
}

Cite This: J. Phys. Chem. Lett. 2020, 11, 2859-2863

Read Online

ABSTRACT: Polycyclic aromatic hydrocarbons (PAHs) play an important role in chemistry both in the terrestrial setting and in the interstellar medium. Various, albeit often inefficient, chemical mechanisms have been proposed to explain $\mathrm{PAH}$ formation, but few yield polycyclic hydrocarbons cleanly. Alternative and quite promising pathways have been suggested to address these shortcomings with key starting reactants including resonance stabilized radicals (RSRs) and $o$-benzyne. Here we report on a combined experimental and theoretical study of the reaction allyl $+o$ benzyne. Indene was found to be the primary product and statistical modeling predicts only $0.1 \%$ phenylallene and $0.1 \%$ 3-phenyl-1-propyne as side products. The quantitative and likely barrierless formation of indene yields important insights into the role resonance stabilized radicals play in the formation of polycyclic hydrocarbons.

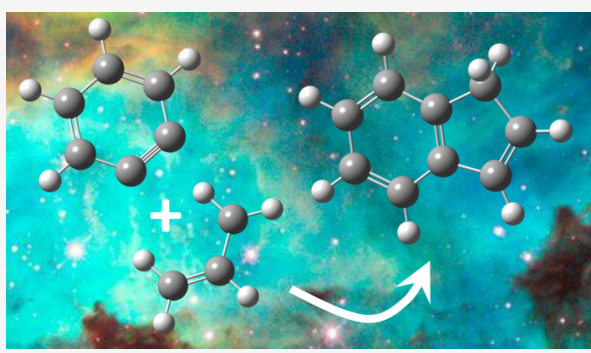

$\mathrm{P}$ olycyclic aromatic hydrocarbons (PAHs) are ubiquitous, both terrestrially ${ }^{1,2}$ and in the interstellar medium. ${ }^{3}$ On Earth, PAHs are formed on a large scale by natural and anthropogenic combustion processes, ${ }^{4}$ while in space, they are believed to be formed in outflows of carbon-rich stars. ${ }^{5}$ Breakthroughs by crossed molecular beam and pyrolysis photoionization mass spectrometry studies have greatly enhanced our understanding of PAH formation..$^{6-10}$ However, many of these mechanisms do not selectively produce polycyclic species but also branched isomers, and/or are inefficient as an entrance barrier limits the reaction rate.

Over the past decades, it has become clear that resonance stabilized radicals (RSRs) are important precursors in PAH formation and soot inception. ${ }^{11,12}$ In RSRs, the delocalized unpaired electron results in resonance structures and increased chemical stability compared to radicals without resonance stabilization. This enhanced stability leads to longer lifetimes and larger number densities in combustion environments. ${ }^{13}$ Several studies have shown that reactions involving RSRs, such as propargyl $\left(\mathrm{C}_{3} \mathrm{H}_{3}{ }^{\bullet}\right)$ and allyl $\left(\mathrm{C}_{3} \mathrm{H}_{5}{ }^{\bullet}\right)$, are crucial in the formation of the first (aromatic) ring. ${ }^{13}$ Furthermore, it was shown that the reaction between the resonance stabilized indenyl radical $\left(\mathrm{C}_{9} \mathrm{H}_{7}{ }^{\circ}\right)$ and the methyl radical $\left(\mathrm{CH}_{3}{ }^{\bullet}\right)$ results in the formation of the smallest $\mathrm{PAH}$, naphthalene. ${ }^{16}$

Recent combustion studies suggest that pentagonal defects are commonly included in the otherwise pristine hexagonal carbon structures found in aromatic molecules. ${ }^{17,18}$ These pentagon-bearing aromatic hydrocarbons have been shown to play a crucial role in the buildup of larger aromatic structures and soot. ${ }^{11}$ Since pentagons are also responsible for the curvature of fullerenes and nanotubes, understanding their formation is clearly crucial to appreciate their role in PAH and soot formation.
ortho-Benzyne has captivated chemists for over 90 years, thanks, in part, to the species having both aryne and biradical character, which in turn affects its reactivity. ${ }^{19,20}$ Yet, its potential to form large aromatic structures in combustion reactions has only recently been recognized. ${ }^{21,22}$ In fact, studies with $o$-benzyne are proving to be increasingly important to investigate, as more research emerges showing the formation of $o$-benzyne in both combustion ${ }^{23}$ and astrochemically relevant environments. $^{24}$ Subsequently, reactions of RSRs with obenzyne represent alternative pathways to polycyclic hydrocarbons. Yet, studies of such reactions are few and far between and limited to only computational works. Propargyl radicals were suggested to yield the indenyl radical when reacting with $o$-benzyne. ${ }^{25}$ Additionally, the cyclopentadienyl radical was computed to yield multiple multiring species by reaction with o-benzyne. ${ }^{26}$

Here we present a combined experimental and computational study of the allyl $+o$-benzyne reaction studied by double imaging photoelectron photoion coincidence spectroscopy (i ${ }^{2}$ PEPICO) on the CRF-PEPICO endstation at the vacuum ultraviolet (VUV) beamline of the Swiss Light Source. ${ }^{27,28}$ A more detailed description of the experimental system is provided in the Supporting Information. The reaction is initiated in a microtubular $\mathrm{SiC}$ reactor that is resistively heated to a temperature of $\sim 1000 \mathrm{~K}$. Allyl ( $4 \mathbf{a}$ and $4 \mathbf{b}$ ) and $o$-benzyne

Received: February 4, 2020

Accepted: March 23, 2020

Published: March 23, 2020 
(2a and $\mathbf{2 b}$ ) are generated by flash pyrolysis of allyl iodide (3) and benzocyclobutenedione (1), respectively (see Schemes 1 and 2). The latter was synthesized according to the procedure described in the Supporting Information.

Scheme 1. Benzocylcobutenedione (1) Pyrolysis to oBenzyne ( $2 a$ and $2 b$ )

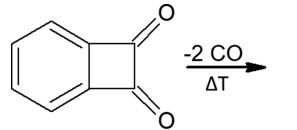

1

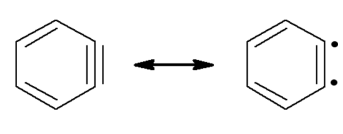

$2 \mathrm{a}$

2b
Scheme 2. Allyl Iodide (3) Pyrolysis to Allyl Radical (4a and 4b)

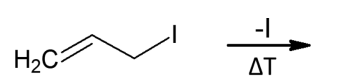

3

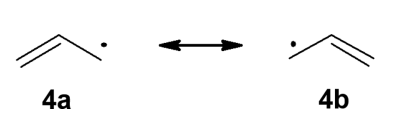

$4 b$
Mass spectra were recorded on the basis of delayed coincidences between electrons and ions. ${ }^{29}$ Figure 1 shows the mass spectra of the two precursors introduced in the microreactor alone and combined.

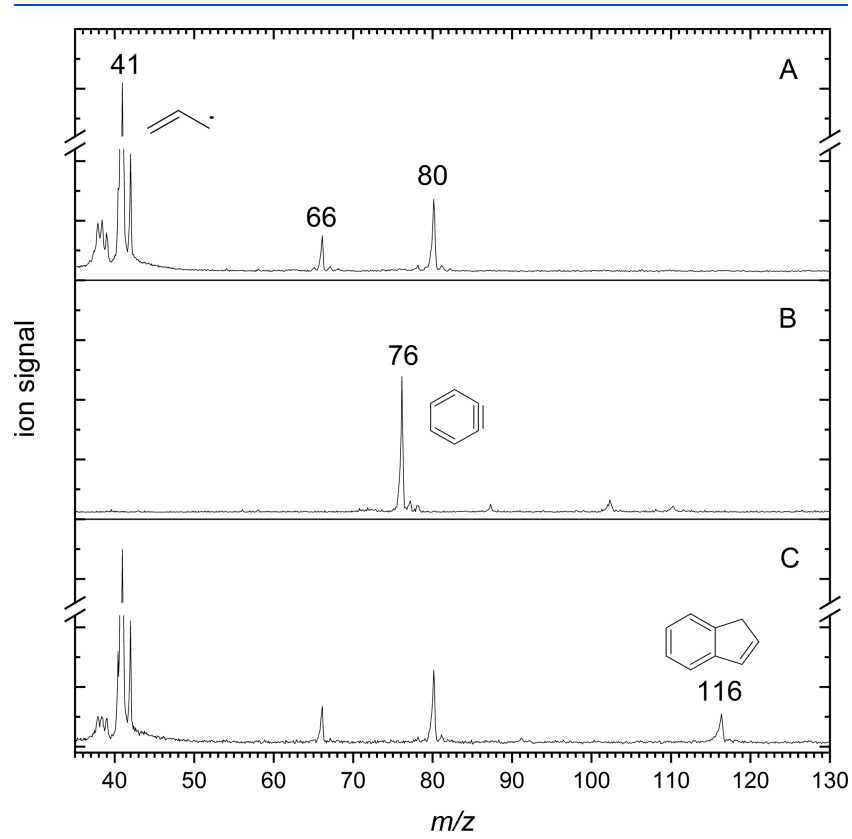

Figure 1. Mass spectrum of (A) allyl iodide (16\% in argon) taken at $9.0 \mathrm{eV}$ and $1045 \mathrm{~K},(\mathrm{~B})$ benzocyclobutenedione (1\% in argon) taken at $9.5 \mathrm{eV}$ and $1080 \mathrm{~K}$, and (C) allyl iodide (16\% in argon) and benzocyclobutenedione ( $1 \%$ in argon) together at $9.0 \mathrm{eV}$ and $1045 \mathrm{~K}$. Masses and structures of key species are shown in the mass spectra.

A detailed description of peak assignments can be found in the Supporting Information and only peaks relevant to the $o$ benzyne + allyl reaction are discussed here. Figure 1A shows a strong signal at $m / z$ 41, which is indicative of efficient conversion of allyl iodide to allyl radical, while Figure $1 \mathrm{~B}$ contains a peak at $m / z 76$, corresponding to o-benzyne. Electron kinetic energy analysis and the time-of-flight mass analysis of the coincident photoion allow us to plot the photoion mass-selected threshold photoelectron spectrum, msTPES, which provides an isomer selective fingerprint to assign the $m / z$ peaks. Contributions of the cis- and trans-1,5- hexadiyne-3-en, which are also $\mathrm{C}_{6} \mathrm{H}_{4}$ isomers, can thus be fully ruled out.

Lastly, Figure 1C shows the presence of a single new peak at $\mathrm{m} / z 116$ associated with the mass corresponding to the addition of allyl to o-benzyne followed by the loss of a hydrogen atom according to Scheme 3.

Scheme 3. o-Benzyne (2) Addition to Allyl Radical (4) Yielding Indanyl Radical Intermediate (5) Followed by Hydrogen Loss To Form Indene (6)

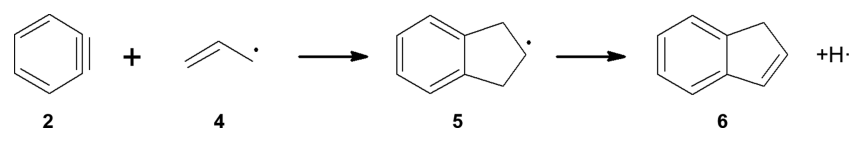

The $m / z 116 \mathrm{~ms}$-TPES was recorded in the 7.6-9.2 eV photon energy range to determine the isomeric composition of the $\mathrm{C}_{9} \mathrm{H}_{8}$ product (Figure 2). The clear vibronic structure

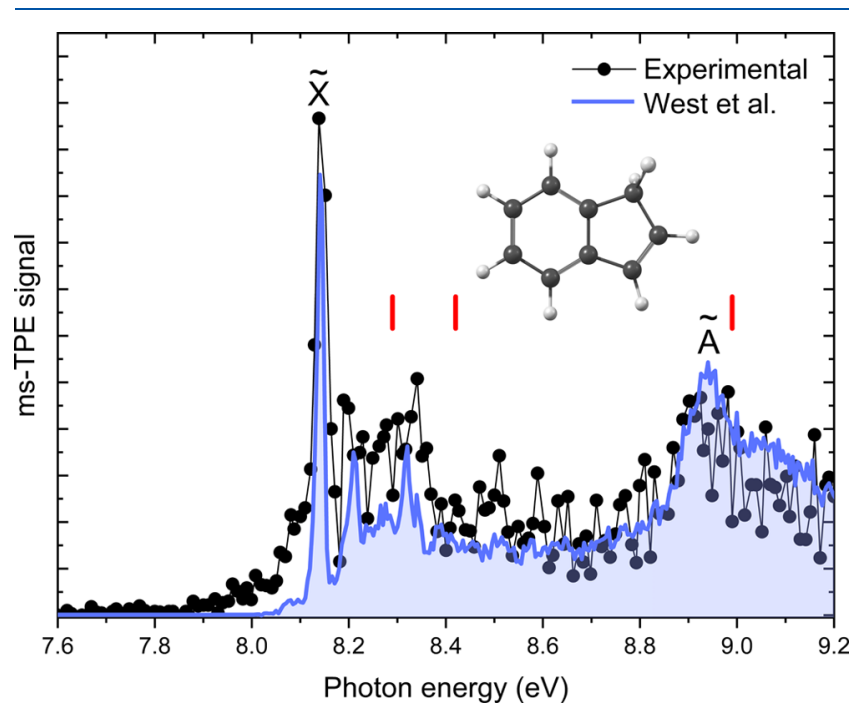

Figure 2. TPES of product at $m / z 116$ taken from 7.6 to $9.2 \mathrm{eV}$ at 930 $\mathrm{K}$ compared to reference TPES of indene provided by West et al. ${ }^{30}$ The red marks indicate the ionization thresholds of three possibly formed $\mathrm{C}_{9} \mathrm{H}_{8}$ isomers.

matches the indene reference TPES recorded by West et al. ${ }^{30}$ at room temperature. The sharp resonance observed at $8.14 \mathrm{eV}$ is in excellent agreement with the indene ionization energy, and the first electronically excited state of the ion is also clearly visible at $8.93 \mathrm{eV}$. The early ms-TPES signal onset before the main resonance is caused by vibrational hot and sequence bands as pointed out by Cunha de Miranda et al. ${ }^{31}$ Ionization energies of three isomeric products that potentially contribute, phenylallene $(8.29 \mathrm{eV}),{ }^{32}$ 3-phenyl-1-propyne $(8.99 \mathrm{eV})$, and 1-phenyl-1-propyne $(8.42 \mathrm{eV})^{33}$ are indicated using red tick marks and do not contribute to the ms-TPES significantly, if at all.

The $\mathrm{C}_{9} \mathrm{H}_{9}$ potential energy surface (PES) was sampled by scanning internal coordinates at the uB3LYP/6-311++G(d,p) level of theory to yield insights into the formation mechanism of the various $\mathrm{C}_{9} \mathrm{H}_{8}$ isomers. G4 calculations were subsequently carried out for the intermediates (INT) and transition states (TS) and a simplified summary of the PES is shown in Figure 3. Using uB3LYP, no entrance barrier to reaction is found, and the allyl radical readily adds to one of the two radical centers of $o$-benzyne, forming INT1. From 


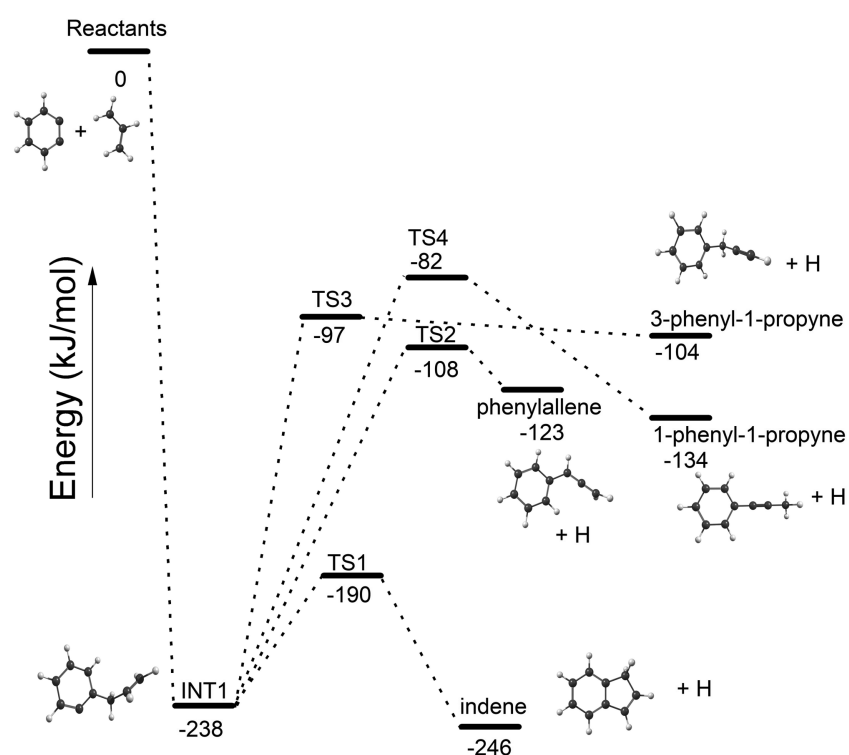

Figure 3. Summary of the $\mathrm{C}_{9} \mathrm{H}_{9}$ potential energy surface with energies calculated at the G4 level of theory. Energies are displayed relative to the reactants and indicated in $\mathrm{kJ} / \mathrm{mol}$.

INT1, ring closure proceeds via allyl rotation and through TS1, which is the rate limiting transition state for the formation of indene. A more detailed figure of the potential energy surface can be found in the Supporting Information. In addition, calculations on the initial reaction to form INT1 were performed using different methods, described in detail in the Supporting Information. From these calculations it was found that the association reaction of allyl with benzyne is likely barrierless, or nearly so, as a negligible barrier cannot be completely ruled out.

Pathways leading to the other potential products, phenylallene, 3-phenyl-1-propyne, and 1-phenyl-1-propyne, were also explored and are summarized in Figure 3 and the Supporting Information. These isomers all fall well below the energy of the reactants and their formation is, thus, energetically allowed. The rate-limiting transitions states are TS2, TS3, and TS4, respectively, which are all substantially $(>82 \mathrm{~kJ} / \mathrm{mol})$ higher in energy than TS1 on the path to indene.

Rice-Ramsperger-Kassel-Marcus (RRKM) theory was employed to estimate the branching over the various products. $^{34}$ Rate constants for crossing the rate limiting barriers for each of the product species are calculated as a function of energy (Figure 4) and a 99.8\% indene, 0.1\% phenylallene, and $0.1 \%$ 3-phenyl-1-propyne branching is predicted at the entrance energy (indicated by the dotted line in Figure 4). The contributions of the fourth isomer, 1phenyl-1-propyne, is found to be negligible. This supports the experimental observation that indene is the primary reaction product.

The bimolecular reaction of $o$-benzyne with allyl is found to produce bicyclic indene exclusively. The facile formation of the five-membered ring species is intriguing in the context of recent findings that pentagon-bearing species are important in both the growth and destruction of PAHs. ${ }^{18,35,36}$ Furthermore, the formation of a polycyclic species from o-benzyne + a RSR reaction confirms the computational findings of Matsugi and Miyoshi $^{25}$ that multiring species are favored over open-chain species in these types of reactions. While estimates of pressure and temperature dependent rate constants are outside the

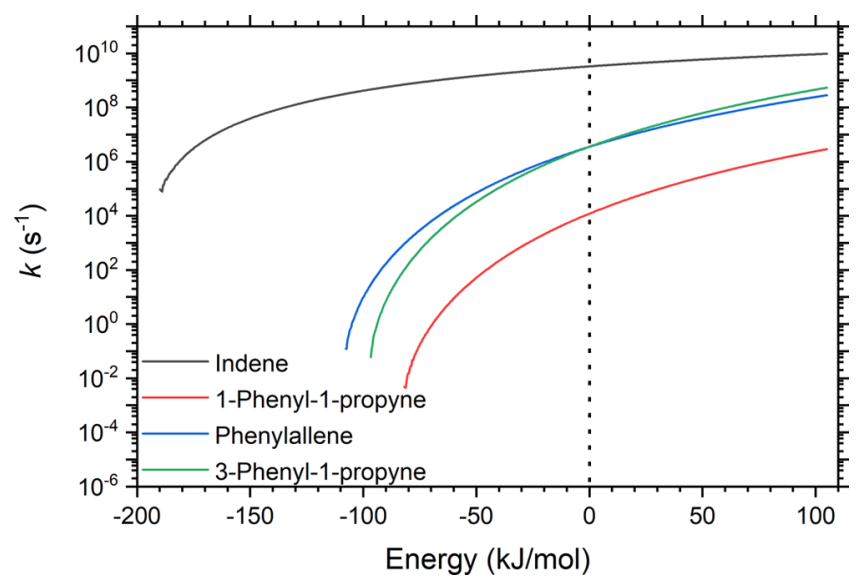

Figure 4. Rates of formation for indene, phenylallene, 1-phenyl-1propyne, and 3-phenyl-1-propyne based on RRKM theory as a function of energy relative to the entrance energy (dotted line).

scope of this Letter, on the basis of the computations, it is clear that the formation rate of INT1 will determine the production of indene for a large range of energies, as the reverse reaction of INT1 toward allyl and o-benzyne is hardly competitive. As a result, indene formation will be governed by collision rate and the impact factor, which while not estimated here, is expected to be high due to $o$-benzyne containing two sites of attack by either of the $\mathrm{CH}_{2}$ groups of allyl.

Such condensation reactions, i.e., those with little to no entrance barrier, may also play an important role in the formation of aromatic molecules in low-temperature environments, such as Titan's atmosphere and in cold molecular clouds. $^{37,38}$ After association, the forward condensation reaction is faster than the dissociation and is made irreversible by the loss of the hydrogen, which also allows thermal stabilization of the product indene. However, at high temperatures, further hydrogen loss from indene yields the indenyl RSR (see Supporting Information). Indenyl plays a critical role in the formation of large aromatic species. ${ }^{16,39}$ This suggests that reactions of $o$-benzyne and RSRs may play a central role in initiating $\mathrm{PAH}$ formation in high temperature environments, such as combustion engines and the outflow of carbon-rich stars, as well.

\section{ASSOCIATED CONTENT}

\section{Supporting Information}

The Supporting Information is available free of charge at https://pubs.acs.org/doi/10.1021/acs.jpclett.0c00374.

Experimental procedures and characterization data for reaction (including ionization energies, mass spectra, ion signals vs pyrolysis temperature, fractional abundance vs temperature, potential energy surfaces and scans, and transition state structures). (PDF)

\section{AUTHOR INFORMATION}

\section{Corresponding Author}

Jordy Bouwman - Laboratory for Astrophysics, Leiden Observatory, Leiden University, 2300 RA Leiden, The Netherlands; (1) orcid.org/0000-0002-3615-1703; Phone: +31(0)715278432; Email: bouwman@ strw.leidenuniv.nl 


\section{Authors}

Morgan N. McCabe - Laboratory for Astrophysics, Leiden Observatory, Leiden University, 2300 RA Leiden, The Netherlands

Patrick Hemberger - Laboratory for Synchrotron Radiation and Femtochemistry, Paul Scherrer Institute, 5232 Villigen, Switzerland; 이이이.org/0000-0002-1251-4549

Engelbert Reusch - Institute of Physical and Theoretical Chemistry, University of Würzburg, D-97074 Würzburg, Germany

Andras Bodi - Laboratory for Synchrotron Radiation and Femtochemistry, Paul Scherrer Institute, 5232 Villigen, Switzerland; (1) orcid.org/0000-0003-2742-1051

Complete contact information is available at: https://pubs.acs.org/10.1021/acs.jpclett.0c00374

\section{Notes}

The authors declare no competing financial interest.

\section{ACKNOWLEDGMENTS}

J.B. acknowledges The Netherlands Organisation for Scientific Research (Nederlandse Organisatie voor Wetenschappelijk Onderzoek, NWO) for a Vidi grant (grant number 723.016.006), which was used to support this work. Furthermore, the authors thank the NWO for the use of the supercomputer facilities at SURFSara (grant no. 17676). The $i^{2}$ PEPICO experiments were performed at the VUV beamline at the Swiss Light Source (Paul Scherrer Institute, 5232 Villigen, Switzerland). P.H. and A.B. gratefully acknowledge funding by the Swiss Federal Office of Energy (BFE Contract Number SI/501269-01). Finally, the authors thank Brandi West for providing the reference TPES of indene.

\section{REFERENCES}

(1) McEnally, C. S.; Pfefferle, L. D.; Atakan, B.; Kohse-Höinghaus, K. Studies of aromatic hydrocarbon formation mechanisms in flames: Progress towards closing the fuel gap. Prog. Energy Combust. Sci. 2006, 32, 247-294.

(2) Richter, H.; Howard, J. B. Formation of polycyclic aromatic hydrocarbons and their growth to soot-a review of chemical reaction pathways. Prog. Energy Combust. Sci. 2000, 26, 565-608.

(3) Tielens, A. G. G. M. Interstellar Polycyclic Aromatic Hydrocarbon Molecules. Annu. Rev. Astron. Astrophys. 2008, 46, 289-337.

(4) Ravindra, K.; Sokhi, R.; Grieken, R. V. Atmospheric polycyclic aromatic hydrocarbons: Source attribution, emission factors and regulation. Atmos. Environ. 2008, 42, 2895-2921.

(5) Cherchneff, I.; Barker, J. R.; Tielens, A. G. G. M. Polycyclic Aromatic Hydrocarbon Formation in Carbon-rich Stellar Envelopes. Astrophys. J. 1992, 401, 269.

(6) Gu, X.; Zhang, F.; Guo, Y.; Kaiser, R. I. Crossed-MolecularBeam Study on the Formation of Phenylacetylene from Phenyl Radicals and Acetylene. Angew. Chem., Int. Ed. 2007, 46, 6866-6869.

(7) Zhang, F.; Kaiser, R. I.; Kislov, V. V.; Mebel, A. M.; Golan, A.; Ahmed, M. A VUV Photoionization Study of the Formation of the Indene Molecule and Its Isomers. J. Phys. Chem. Lett. 2011, 2, 17311735.

(8) Kaiser, R. I.; Parker, D. S. N.; Mebel, A. M. Reaction Dynamics in Astrochemistry: Low-Temperature Pathways to Polycyclic Aromatic Hydrocarbons in the Interstellar Medium. Annu. Rev. Phys. Chem. 2015, 66, 43-67.

(9) Parker, D. S. N.; Kaiser, R. I.; Kostko, O.; Ahmed, M. Selective Formation of Indene through the Reaction of Benzyl Radicals with Acetylene. ChemPhysChem 2015, 16, 2091-2093.
(10) Yang, T.; Troy, T. P.; Xu, B.; Kostko, O.; Ahmed, M.; Mebel, A. M.; Kaiser, R. I. Hydrogen-Abstraction/Acetylene-Addition Exposed. Angew. Chem., Int. Ed. 2016, 55, 14983-14987.

(11) Johansson, K. O.; Head-Gordon, M. P.; Schrader, P. E.; Wilson, K. R.; Michelsen, H. A. Resonance-stabilized hydrocarbon-radical chain reactions may explain soot inception and growth. Science 2018, 361, 997-1000.

(12) Miller, J. A.; Klippenstein, S. J. The Recombination of Propargyl Radicals and Other Reactions on a $\mathrm{C}_{6} \mathrm{H}_{6}$ Potential. J. Phys. Chem. A 2003, 107, 7783-7799.

(13) Hansen, N.; Miller, J. A.; Klippenstein, S. J.; Westmoreland, P. R.; Kohse-Höinghaus, K. Exploring formation pathways of aromatic compounds in laboratory-based model flames of aliphatic fuels. Combust., Explos. Shock Waves 2012, 48, 508-515.

(14) Bouwman, J.; Bodi, A.; Oomens, J.; Hemberger, P. On the Formation of Cyclopentadiene in the $\mathrm{C}_{3} \mathrm{H}_{5} \bullet+\mathrm{C}_{2} \mathrm{H}_{2}$ reaction. Phys. Chem. Chem. Phys. 2015, 17, 20508-20514.

(15) Miller, J. A.; Klippenstein, S. J.; Georgievskii, Y.; Harding, L. B.; Allen, W. D.; Simmonett, A. C. Reactions between ResonanceStabilized Radicals: Propargyl + Allyl. J. Phys. Chem. A 2010, 114, 4881-4890.

(16) Zhao, L.; Kaiser, R. I.; Lu, W.; Xu, B.; Ahmed, M.; Morozov, A. N.; Mebel, A. M.; Hasan Howlader, A.; Wnuk, S. F. Molecular mass growth through ring expansion in polycyclic aromatic hydrocarbons via radical-radical reactions. Nat. Commun. 2019, 10, 3689.

(17) Commodo, M.; Kaiser, K.; De Falco, G.; Minutolo, P.; Schulz, F.; D'Anna, A.; Gross, L. On the early stages of soot formation: Molecular structure elucidation by high-resolution atomic force microscopy. Combust. Flame 2019, 205, 154-164.

(18) Schulz, F.; Commodo, M.; Kaiser, K.; Falco, G. D.; Minutolo, P.; Meyer, G.; D'Anna, A.; Gross, L. Insights into incipient soot formation by atomic force microscopy. Proc. Combust. Inst. 2019, 37, 885-892.

(19) Wenk, H. H.; Winkler, M.; Sander, W. One Century of Aryne Chemistry. Angew. Chem., Int. Ed. 2003, 42, 502-528.

(20) Wentrup, C. The Benzyne Story. Aust. J. Chem. 2010, 63, 979986.

(21) Comandini, A.; Abid, S.; Chaumeix, N. Polycyclic Aromatic Hydrocarbon Growth by Diradical Cycloaddition/Fragmentation. J. Phys. Chem. A 2017, 121, 5921-5931.

(22) Hirsch, F.; Reusch, E.; Constantinidis, P.; Fischer, I.; Bakels, S.; Rijs, A. M.; Hemberger, P. Self-Reaction of ortho-Benzyne at High Temperatures Investigated by Infrared and Photoelectron Spectroscopy. J. Phys. Chem. A 2018, 122, 9563-9571.

(23) Matsugi, A. Thermal Decomposition of Benzyl Radicals: Kinetics and Spectroscopy in a Shock Tube. J. Phys. Chem. A 2020, 124, 824-835.

(24) Zhang, F.; Parker, D.; Seol Kim, Y.; Kaiser, R. I.; Mebel, A. M. On the Formation of Ortho-benzyne $\left(o-\mathrm{C}_{6} \mathrm{H}_{4}\right)$ Under Single Collision Conditions and its Role in Interstellar Chemistry. Astrophys. J. 2011, $728,141$.

(25) Matsugi, A.; Miyoshi, A. Computational study on the recombination reaction between benzyl and propargyl radicals. Int. J. Chem. Kinet. 2012, 44, 206-218.

(26) Comandini, A.; Brezinsky, K. Radical/pi-Bond Addition between o-Benzyne and Cyclic C5 Hydrocarbons. J. Phys. Chem. A 2012, 116, 1183-1190.

(27) Sztáray, B.; Voronova, K.; Torma, K. G.; Covert, K. J.; Bodi, A.; Hemberger, P.; Gerber, T.; Osborn, D. L. CRF-PEPICO: Double velocity map imaging photoelectron photoion coincidence spectroscopy for reaction kinetics studies. J. Chem. Phys. 2017, 147, 013944.

(28) Bodi, A.; Hemberger, P.; Gerber, T.; Sztáray, B. A new double imaging velocity focusing coincidence experiment: i2PEPICO. Rev. Sci. Instrum. 2012, 83, 083105.

(29) Osborn, D. L.; Hayden, C. C.; Hemberger, P.; Bodi, A.; Voronova, K.; Sztáray, B. Breaking through the false coincidence barrier in electron-ion coincidence experiments. J. Chem. Phys. 2016, $145,164202$. 
(30) West, B.; Sit, A.; Bodi, A.; Hemberger, P.; Mayer, P. M. Dissociative Photoionization and Threshold Photoelectron Spectra of Polycyclic Aromatic Hydrocarbon Fragments: An Imaging Photoelectron Photoion Coincidence (iPEPICO) Study of Four Substituted Benzene Radical Cations. J. Phys. Chem. A 2014, 118, 11226-11234.

(31) Cunha de Miranda, B. K.; Alcaraz, C.; Elhanine, M.; Noller, B.; Hemberger, P.; Fischer, I.; Garcia, G. A.; Soldi-Lose, H.; Gans, B.; Vieira Mendes, L. A.; et al. Threshold Photoelectron Spectroscopy of the Methyl Radical Isotopomers, $\mathrm{CH}_{3}, \mathrm{CH}_{2} \mathrm{D}, \mathrm{CHD}_{2}$ and $\mathrm{CD}_{3}$ : Synergy between VUV Synchrotron Radiation Experiments and Explicitly Correlated Coupled Cluster Calculations. J. Phys. Chem. A 2010, 114, 4818-4830.

(32) Runge, W.; Kosbahn, W.; Kroner, J. The Molecular Structure of Allenes and Ketenes II [1] Photoelectron Spectra, Absorption Spectra, and CNDO/S-Calculations of Phenyl and Methyl Substituted Allenes. Ber. Bunsengesell. Phys. Chem. 1975, 79, 371-381.

(33) Elbel, S.; Lienert, K.; Krebs, A.; Dieck, H. T. Photoelektronenspektren von Phenylethinen, I. Phenylethin - Mustersonde für Substituenteneffekte. Liebigs Ann. Chem. 1981, 1981, 1785-1797.

(34) Sztáray, B.; Bodi, A.; Baer, T. Modeling unimolecular reactions in photoelectron photoion coincidence experiments. J. Mass Spectrom. 2010, 45, 1233-1245.

(35) Bouwman, J.; de Haas, A. J.; Oomens, J. Spectroscopic evidence for the formation of pentalene ${ }^{+}$in the dissociative ionization of naphthalene. Chem. Commun. 2016, 52, 2636-2638.

(36) de Haas, A. J.; Oomens, J.; Bouwman, J. Facile pentagon formation in the dissociation of polyaromatics. Phys. Chem. Chem. Phys. 2017, 19, 2974-2980.

(37) Waite, J. H.; Young, D. T.; Cravens, T. E.; Coates, A. J.; Crary, F. J.; Magee, B.; Westlake, J.; et al. The Process of Tholin Formation in Titan's Upper Atmosphere. Science 2007, 316, 870-875.

(38) McGuire, B. A.; Burkhardt, A. M.; Kalenskii, S.; Shingledecker, C. N.; Remijan, A. J.; Herbst, E.; McCarthy, M. C. Detection of the aromatic molecule benzonitrile $\left(\mathrm{c}^{-} \mathrm{C}_{6} \mathrm{H}_{5} \mathrm{CN}\right)$ in the interstellar medium. Science 2018, 359, 202-205.

(39) Hansen, N.; Kasper, T.; Klippenstein, S. J.; Westmoreland, P. R.; Law, M. E.; Taatjes, C. A.; Kohse-Höinghaus, K.; Wang, J.; Cool, T. A. Initial Steps of Aromatic Ring Formation in a Laminar Premixed Fuel-Rich Cyclopentene Flame†. J. Phys. Chem. A 2007, 111, 40814092. 